Article

\title{
Implementation and Evaluation of Nodal Distribution and Movement in a 5G Mobile Network
}

\author{
Dmitry Baranov (D), Alexandr Terekhin (D, Dmitry Bragin (D) and Anton Konev *(D) \\ Faculty of Security, Tomsk State University of Control Systems and Radioelectronics, Tomsk 634034, Russia; \\ bda@csp.tusur.ru (D.B.); tao@csp.tusur.ru (A.T.); bds@csp.tusur.ru (D.B.) \\ * Correspondence: kaa1@fb.tusur.ru
}

check for updates

Citation: Baranov, D.; Terekhin, A.; Bragin, D.; Konev, A. Implementation and Evaluation of Nodal Distribution and Movement in a $5 \mathrm{G}$ Mobile Network. Future Internet 2021, 13, 321. https://doi.org/10.3390/fi13120321

Academic Editors: Ammar Muthanna and Mohammed Abo-Zahhad

Received: 6 December 2021

Accepted: 17 December 2021

Published: 20 December 2021

Publisher's Note: MDPI stays neutral with regard to jurisdictional claims in published maps and institutional affiliations.

Copyright: (C) 2021 by the authors. Licensee MDPI, Basel, Switzerland. This article is an open access article distributed under the terms and conditions of the Creative Commons Attribution (CC BY) license (https:/ / creativecommons.org/licenses/by/ $4.0 /)$.

\begin{abstract}
The determining factor in the accelerated pace of informatization is the increase in the speed and reliability of data transmission networks. In this regard, new and existing standards are developed and modernized. A lot of organizations are constantly working on the development and implementation of new generation communication networks. This article provides an overview of available software solutions that allow us to investigate and evaluate the behavior of data networks. In particular, tools suitable for mobile communication systems were determined, having sufficient built-in functionality and allowing us to add our own implementations. NS3 has been chosen as a suitable network simulator. Apart from the review, a solution for this tool was developed. It allows estimating the reliability of data transmission from the start movement of a network node at all times during its removal from a base station.
\end{abstract}

Keywords: 5G; networks; security; reliability; NS3; Okumura-Hata

\section{Introduction}

The process of global informatization of contemporary society brings the necessity to increase the operation speed and reliability of data-transmission networks. In particular, the 3GPP consortium has an active influence on mobile communications, developing and updating new and existing standards. As a result, continuous work is underway to develop and implement new generation communication networks and methods of combating information security threats in them.

The continuous growth and modernization of the digital economy, under the prodigious influence of artificial intelligence and Big Data analysis, in the contemporary world has led to the need for a transition to a new generation of communication networks, the socalled "fifth generation" or 5G. 5G networks significantly expand the limited functionality of previous generations of mobile networks. The main functional features of 5G networks are as follows:

- $\quad$ Enhanced mobile broadband (eMBB);

- $\quad$ Ultra Low Latency Reliable Communication (ULLRC);

- $\quad$ massive Machine Type Communication, Massive IoT/IIoT (mMTC).

Simulation modeling is in active use for research on factors weighting the quality, speed and behavior of nodes in various scenarios occurring in mobile networks. Due to the progression of such an approach, it is customary to simulate the process of data transmission before hardware-software implementation nowadays. Additionally, many of these tools can be applied both to test network technologies and validate network deployments in the real world.

\subsection{Motivation and Contribution}

There is strong interest in the study of factors precisely influencing the qualitative indicators of communication. Therefore, as early as the simulation, it is also necessary 
to generate noise and study its effect on the system. It is an intrinsic element of noise immunity in the field of information security and telecommunications networks. The motive is behind the shaping of network simulation scenarios. They demonstrate the process of influencing not only network subscribers, but also sources of interference.

There are situations when the built-in set of available modules, designed to generate simulation scenarios, prevents full consideration of aspects in this area. Therefore, the developers assign the task of finalizing and introducing additional implementations. Therefore, the overview part presents both the existing ready-made network tools and projects on research related to the growth of mobile $5 \mathrm{G}$ networks. In addition, algorithms for the behavior of network subscribers were implemented, and results were obtained that allow one to determine the interference pattern.

\subsection{Organization}

The rest of the paper is organized as follows. Section 2 includes a review of available network simulators. Section 3 includes a review of actual NS3 network simulator projects, which includes 5G mobile networks and their improvement or research. Section 4 presents development of distribution and movement functions. Section 5 presents results and analysis, and Section 6 is discussion and conclusions.

\section{Review of Simulators}

The hardware and software implementation of ready-made solutions in the field of testing mobile networks, taking into account the behavior of its nodes in various conditions, is not rational both from an economic point of view and from the point of view of provision guaranteed reliability data transmission. As a consequence, it is customary to perform software simulations of processes occurring in mobile networks. Given this, a review of the available ready-made software solutions that would maximally cover the issues raised above was carried out. These questions can be formulated as follows:

- Provision and modernization of reliable data transmission in continuously evolutionary networks;

- The ability to scale the topology and behavior of nodes in the network;

- The possibility of implementing threats and countermeasures to them with the use of methods of information security of telecommunication networks (these criteria are described in more detail in the works [1,2]);

- Using the maximum number of possible layers of the data communication protocol stack.

As a review, all the proposed solutions with implemented simulation are divisible into software use of simulators and emulators. For the reasons already shown earlier, these are the arising hardware implementation difficulties.

A simulator is a part of the software that simulates the topology of a network. The simulated network devices are not real devices and cannot carry real network traffic. The network devices in the simulator are limited by the commands and functions programmed into them. For this reason, many of the additional features presented on real network devices are absent in the simulated analogues. The main advantage of simulators is in their less demanding computational resources.

In its turn, an emulator is software that works with virtual network devices. Emulators virtualize real network devices, so the behavior of virtual network devices reflects the behavior of real-world physical devices.

\subsection{Cisco Packet Tracer}

Cisco Packet Tracer is a Cisco visual modeling tool that simulates network topologies of routers, switches, firewalls, etc. Originally, the Packet Tracer was developed as a study guide for the Cisco Networking Academy, but it is usable as a simulator for students education. It is necessary to create a free account of Cisco Networking Academy to get started with it [3,4]. 
An essential advantage of this tool is in its cross-platforming. Additionally, it includes a rich set of simulation devices and provides many variants to connect these devices to each other. Special attention is given to sufficiently flexible device configuration and real-time operation. The functional graphical user interface (GUI) is also a significant advantage of this emulator. The GUI allows us to configure, check and troubleshoot network device interactions in a convenient way.

From the information security point of view, this tool is suitable because it gives developers a separate direction for implementing attacks and ways to combat them. However, the disadvantage of this approach is that all algorithms are implemented at the level of computer networks and high levels of the OSI network model.

\subsection{Mininet}

Mininet is a computer network emulator. Herein, computer network represents simple computers-hosts, switches, and OpenFlow controllers. It is most often used to learn the basics of working with SDN networks (Open VSwitch). The main advantage of the tool is in simple syntax using a command interpreter. It allows the deployment ofnetworks of an arbitrary number of hosts and switches in different topologies within a single virtual machine. It may be possible to change the network configuration of each network node, use the built-in operating system, standard utilities, and get access to the terminal. Additionally, it may be possible to add various rules and route traffic upon the network [5].

The negative side of this emulator is the the safety issue, since the developers themselves do not cover this aspect. The implementation of attacks and methods combating them depends on the knowledge and capabilities of the users who will use this emulator. It is also limited only by the protocols of computer networks at the high levels of the network model.

\subsection{CORE}

Common Open Research Emulator (CORE) is an emulator of networks with a graphical interface, representing itself as the advanced upgrade of the IMUNES product. This tool has the same advantages and disadvantages as Mininet [6,7].

\subsection{Marionnet}

Marionnet is a project based on teaching principles in the field of networking. The advantages of this emulator are the existing devices set (switches, computers, routers) and the simple graphical interface. However, information security issues for the possibility of own implementations remain open.

\subsection{GNS3}

GNS3 is a free graphical network simulator. It is based on the client-server principle. This tool is open source, and it is used for network emulation and virtualization. The simulator platform is based on the Python programming language emulating Cisco software and hardware. GNS3 supports a wide range of virtual network devices from various network equipment vendors [8,9]. It allows using a convenient template manager, making it easier to add and edit network devices to the network.

GNS3 is a client/server application. A client application is intended for modeling. A server application provides the correct operation of the simulator integrally. A client application should be deployed on a local computer. A server application may be deployed on the same computer as a client application and represented as a virtual machine or on a separate dedicated server, providing an opportunity for remote access to a client application. The connection between the server and the client needs to configure after installing the required simulation components. As a result of the performed operations, it is possible to create network topologies using the client software that can be processed on the server. 
GNS3 has its own community of developers and users. Feedback created by a group of like-minded people who want to help others learn, work, and fix bugs in software implementations is an essential advantages.

A definitive advantage of the emulator is detailed documentation with illustrations for novice users. It is available to observe guides for advanced configuration of both individual network devices and the server-side features. Each virtual network device is an independent network object whose starting or stopping does not affect the operation of another unrelated node. The emulator supports Ethernet connections between network devices and also allows serial connections between devices that support the corresponding modules.

The main disadvantage of GNS3 lies in the fact that the user needs to create your own software images of network devices for emulation, which are optionally paywall. Therefore, the deployment of GNS3 is contingent on personal or commercial use. Additionally, the developers do not consider security issues as one of the main areas. This emulator allows using all implementations present in Cisco Packet Tracer together with it, but requires the availability of the necessary images of operating systems and devices.

\section{6. $E V E-N G$}

EVE-NG (Emulated Virtual Environment Next Generation) is a multi-user virtual network emulator developed for individuals and small businesses [10]. The company offers two major implementations of the simulator: free and professional paid editions, which immediately gives an understanding of the advantages and disadvantages of the software. However, according to the developers, the free version is suited for educational purposes.

The EVE-NG client is a core technical feature setting this emulator apart from the rest. The emulator uses a browser for designing, connecting and managing network topologies. It eliminates the download and installation of individual applications as an addition to the server for virtualization, connection and configuration of network devices. The optimized browser runtime is straightforward when working with large topologies.

EVE-NG allows changing topologies of a network throughout their active execution that saves time when working with nodes whose execution is a resource-consuming operation.

Due to the comparatively recently release of the emulator, the EVE-NG documentation consists of a number of shortcomings. As a result, it can cause difficulties during its usage. Missing ready-made software images in the emulator is also a problem. Therefore, using an emulation through EVE-NG needs to have licensed access to software images of network devices.

One of the features of this product is the user knowledge required working with SSH and experience in the Linux shell for the import and implementation of network devices. As for the issue of information security, the field of application both in learning and in real-world tasks execution is full-solved using this emulator.

The main disadvantage of the EVE-NS, the same as the Cisco Packet Tracer, is in the absence of support physical and data link layers or supports them partly.

\subsection{NS3}

NS3 is a network simulator for discrete event simulation. It is free software under the GNU GPLv2 license. This tool is directed to research applications, as well as for educational purposes $[11,12]$. NS3 has the flexibility and speed of simulation scenarios due to the using $\mathrm{C}++$ as a master programming language. At the same time, it supports the Python language, which gives a lower learning curve for new users using this simulator. Each of these programming languages allows describing the behavior of telecommunication systems.

The NS3 gives the opportunity to developers to build models of any topology and complexity. Along with this, a simulator gives an opportunity to implement, modify and supplement both existing models and modules with the user's own developments. Disadvantages of the NS3 are missing of a fully supported IDE and also missing a builtin graphical interface. However, there are ready-made third-party implementations for visualizing the behavior of models. 
Models of wireless network types have been developed and built into NS3, which allow modeling with moving objects in three-dimensional space. Particular attention is given to the detailed use of the physical and link layer implementations of the OSI network model, which opens the way for many opportunities for research in this area.

The development of the NS3 project does not stop. Updates with the correction of existing shortcomings and the implementation of new standards represented as separate modules or separate products are continuously released. There is also built-in support for distributed computing, and it continuous modernizes, which further accelerates the simulation process.

From the point of information security view, the project developers focus on the reliability of data transmission, leaving these issues of secondary importance. However, the community supports both the implementation and method of combating attacks at the high layers of the OSI model. Most often, the simulator used is the general source of traffic generation and behavior of a node within the network. Specified attention to the implementation of the physical and link layers opens the way for opportunities in security research.

\subsection{Comparison of Network Simulators}

Some of parameters for comparison of network simulators are presented in the Table 1. For a convenient understanding of the table parameters, filled circles have been introduced. An empty circle means there is no current parameter at all. A half-filled circle indicates a partial presence of the parameter in the network tool. A filled circle indicates the complete presence of the parameter. The abbreviations for parameter names are presented in the "Abbreviations" block.

Table 1. Comparison of network simulators.

\begin{tabular}{|c|c|c|c|c|c|c|}
\hline Parameter & Cisco PT & Mininet & Core & GNS3 & EVE-NG & NS3 \\
\hline Free & 0 & O & O & O & O & 0 \\
\hline GUI & 0 & 0 & 0 & 0 & 0 & 0 \\
\hline Cross pl. & 0 & 0 & 0 & 0 & 0 & 0 \\
\hline Realtime & 0 & 0 & 0 & 0 & 0 & 0 \\
\hline WL pr. & $\bigcirc$ & $\bigcirc$ & $\bigcirc$ & $\bigcirc$ & 0 & 0 \\
\hline Phy layer & $\bigcirc$ & 0 & $\bigcirc$ & 0 & 0 & 0 \\
\hline Security & 0 & 0 & 0 & 0 & 0 & 0 \\
\hline Doc. & 0 & 0 & 0 & 0 & 0 & 0 \\
\hline
\end{tabular}

Based on the results obtained, it was decided to use the NS-3 network simulator for further research work. Such indicators as free, availability of ready-made wireless modules, and attention to the physical layer played an important role in choosing a network simulator.

\section{Review of NS3 Modules}

The review of available ready-made solutions in the network technologies area and raised research questions showed that the most suitable solution is a network simulation of discrete events NS3. Primary advantages of the NS3 are the availability of ready-made solutions in wireless networks of information transmission, the flexibility of writing scripts that provide detailed information at low levels of the protocol stack, the ability to write user's own software solutions, continuous support in revision and the possibility adding of new modules.

In this regard, the available solutions in the new-generation mobile networks area and projects that actively gain popularity in researches using the NS3 network simulator were reviewed. One of the revolutionary projects in the field of new generation mobile networks is the implementation of a large separate module that appeared comparatively recently for the integration of millimeter waves in $5 \mathrm{G}$ networks [13-15]. As the developers 
of this solution note, the increasing demand for speed and reliability of data processing causes an urgent transition to new frequency ranges and the rising of new problems as a consequence [16]. It is worth remarking that the initiative group is constantly working upon this module releasing updates and fixing problems in the implementation, thereby opening up new opportunities for many researchers [17,18].

Another independent project using NS3 for 5G networks is the 5G Complete Network Platform [19]. The developers show their view on the implementation of such mobile networks by covering all aspects of $5 \mathrm{G}$ according to the specification. The basis of this module is the division of the code execution logic both into levels and into so-called cases. It provides an encapsulated environment for writing and customizing simulation scripts. It is worth remarking that there is no fully ready-made implementation that takes catering to the needs of each and everyone, but the developers of this module are actively working on updates and bug fixes.

Besides free-standing projects implementing huge functionalities, the modernization of these projects by third-party developers is observed. Thus, one of the ways of multiplexing numerologies with frequency division for the 5G NR module was added and evaluated [20-22]. This solution expands the existing functionality, revealing the other side of the mobile network's behavior.

5G mmWave project serves as another example of expanding the power consumption capabilities of user equipment in 5G networks through improved module integration, including more complex algorithms of energy resources spending [23-25]. The developers gave special attention to the analysis of power consumption in both uninterruptible and interrupt packet transmission processes.

A module called as improved MAC level $[26,27]$ is a significant extension of the 5G NR project existing functional for the MAC level. Based on the success of the LTE and mmWave projects, the developers made functional comparable with the specification and modernized existing schedulers interfaces by that they afforded to use resource distribution at the symbol level.

As noted above, upgrading of existing algorithms is a frequent occurrence when conducting projects in this area. The development of a module for stable and equal quantization of notifications in $5 \mathrm{G}$ mobile networks was no exception. Based on the requirement for a high quality of service in the mobile networks, the developers have changed the existing QCN algorithm, calling it more "honest" and "fair" [28]. The main feature of this approach is the existence of a notification that displays when the standard QCN is overloaded.

Another option for researching the NS3 network simulator usage is module integration with a novel technology (which has no implementation yet). So the development group added FD-MIMO technology to the 5G NR module [29]. Analyzing both the situation and the behavior of the added technology has shown that FD-MIMO overcomes several practical problems such as interference and hardware constraints.

Besides the introduction of new modules, the scientific community analyze existing tools. This way, while analyzing the algorithm for separating a $5 \mathrm{G}$ mobile network based on priority, it was noticed that the goals of this approach, in particular improving performance, security, flexibility and monitoring of networks, are achieved by dramatically increasing latency and reducing work efficiency [30]. The obtained using the simulator results show the need to modernize the existing algorithms.

The increasing attention to neural networks attracts scientists to implement and process data while working with network simulators. Therefore, NS3 is most often used as a traffic generator or a tool for downloading large volumes of data, which is a viable option for neural network models training. For instance, the article [31] analyzes using machine learning to predict channels of the $5 \mathrm{G}$ network of the module mmWave. The presented results show that it is possible to detect a higher percentage of congestion situations using machine learning, opening up opportunities to modernize protocols for future standards. 
The project using adaptive transmission of 3D-video when exchanging data in 5G mobile networks [32] uses an analogous principle working with the NS3. The developers note that the simulator has a ready-made module and affords a convenient way to generate data planned to process by neural networks in the future.

Additionally, Support Vector Machine applies to coordinate self-organizing networks towards optimization and analysis of throughput in mobile networks [33,34]. As in the previous, this project uses the network simulator as a data generator. The exception is in analyzing both $4 \mathrm{G}$ and $5 \mathrm{G}$ networks by comparative between performance and efficiency.

When different networks types interact with each other, the analysis of the behavior of their models implemented with the simulator is also used. For instance, the project to performance evaluation of 5G access technologies and the SDN transport network [35] analyses traffic carried out during transmission in mobile networks of the 4th and 5th generations to the transport SDN network. The estimation results showed that eMBB traffic transmitted by $5 \mathrm{G}$ networks is more suitable for interacting with SDN than $4 \mathrm{G}$ networks, and, also, the transition to a new mobile network generation makes absolute sense from the point of this issue view.

A common enough solution is the issue of virtualization and containerization of services in 5th generation mobile networks. For instance, the analysis and improvement of the existing functionality to implement the container LTE network in 5G through incipient modules is in several projects all at once [36-38]. Additionally, the process of integrating the algorithms for the implementation of virtual small base stations with the possibility of dynamic selection of the more suitable one occurs in the projects. Estimating the behavior of these manipulations in all projects became possible using ready-made modules and scripts of the NS3 network simulator.

There are difficulties with the load on protocol interaction for sending packets when the simulator is used as receiver traffic of 5G mobile networks. It is due to a continuing synchronous increase in the number of intelligent applications and user traffic. In this regard, the development group presented a module with a virtualized and programmable EPC architecture (NOS-EPC) [39,40]. It is supposed that this solution will have flexibility and scalability when other networks interact with the 5G mobile network. The load on this module was checked using the loaded traffic obtained by executing simulation scenarios in the NS3 simulator.

Additionally, the NS3 network simulator is applied for satellite systems development when interacting with wireless networks, including mobile. The proposing solution within the development of the SANSA project is based on the satellite component integrating into the terrestrial network under requirements of traffic received with this network simulator. Additionally, weather conditions influence the transmission process when using satellite equipment in new generation networks. The study of this influence on satellite communications was carried out by creating scenarios for modeling and generating traffic using the NS3 network simulator [41-43].

When carrying out a study on the subject of noise immunity in a wireless network, a detailed consideration of the calculation of the interaction between signals plays an important role. Therefore, based on the studied projects in NS-3, it was decided to use the mmWave project to create a $5 \mathrm{G}$ network model. This project is focused on building 5 th generation networks and has functionality with detailed implementation of the physical channel. The implementation makes it possible to sufficiently fully consider the interaction of signals, in particular, their interference. Interference, in turn, affects the quality of the transmission channel. This is an important aspect when considering networks from an information security point of view.

\section{Implementation of Distribution and Movement}

Based on the above review on using the NS3 network simulator in various fields, it can be concluded that the simulator developers and the initiative groups of developers offer solutions for the modernization and implementation of novel standards, but the security 
issue remains open. Our own implementations to analyze 5G mobile networks in the field of interference immunity have been developed within this article.

It was necessary to form a network model to study the compliance with the criteria for the integrity and availability of information within the framework of this work. The basis of the model was the algorithms for the distribution and movement of nodes. The amount of nodes and their position significantly influences the interfering frequency and channel quality in common.

In this regard, the following tasks were set:

- Developing algorithm of the distribution of $n$ network nodes in a defined area;

- Developing an algorithm of subscriber node movement based on their equidistant from a base station.

The optimal planning of device distribution within a network, nodes movement simulation and analysis of noise immunity throughout the entire process of device interaction is of particular importance when organizing mobile networks. The main task of the models is to determine and predict the parameters of the functioning of a mobile network, such as traffic intensity, delays and packet loss in the channel. For this reason, a subscriber distributing algorithm was implemented, demonstrating the behavior of the signal-to-noise ratio at the initial stage of modeling.

The first step in the distributing algorithm is the definition of a rectangular area. There is a need to set positions of base stations firstly to do this. They will define the area based on their index in the software environment. Further, the area of the distribution of the associated subscribers is set for each base station. This area is bounded by two radii that form a ring. The block scheme of the distribution is shown in Figure 1. As input parameters set following:

- Position of the subscriber's base station;

- Height of the subscriber's antenna;

- Small and large radiuses of the area.

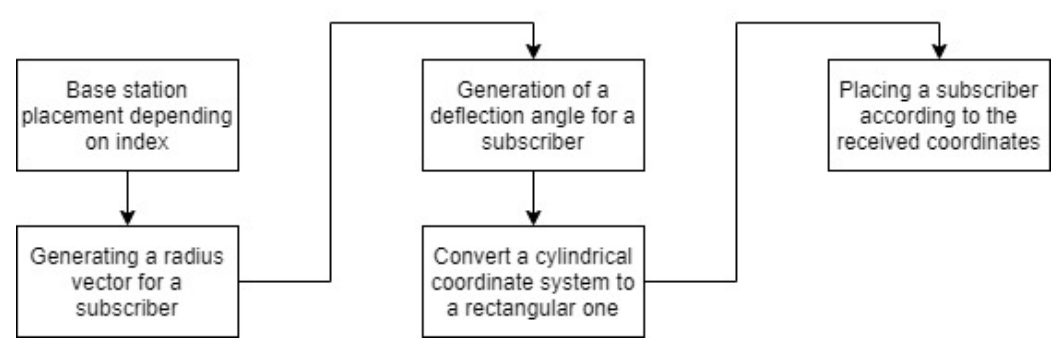

Figure 1. Block scheme of the distribution.

This function determines the distance between the subscriber and the base station and the angle. The base coordinate system in the NS-3 modeling environment is a Cartesiancoordinate system. Therefore, it is necessary to convert the obtained coordinates from cylindrical to Cartesian. As a result, the function returns the position as an object of the Vector class. The full implementation of the function is shown in Algorithm 1.

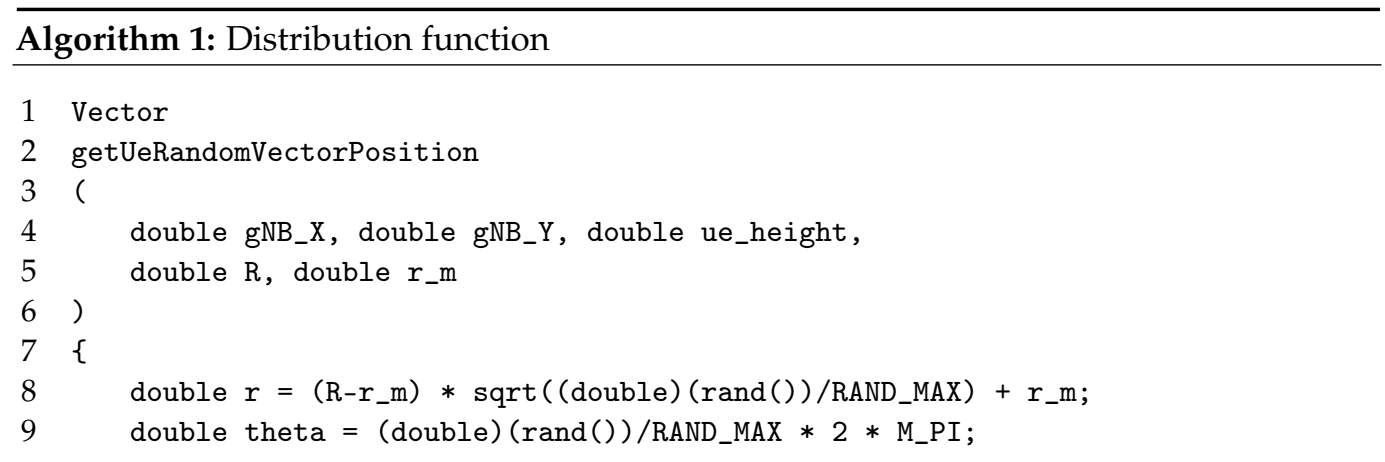




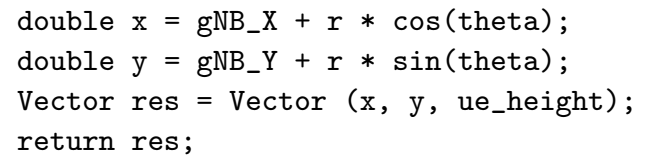

The interference pattern should be changed when the subscribers move. The effect of reducing the SINR indicator should appear when an object moves away from the base station and when it is near other objects in the system. It indicates a deterioration in communication performance. On this basis, the packet loss increases and, consequently, the BLER parameter increases.

A solution, where subscribers moved in a straight line away from the base station, was implemented to observe this event. The function describing the trajectory of subscribers' movement is shown in Figure 2. The linear function (Function 1), set by the positions of the subscriber and the base station, is used to determine the trajectory.

$$
f(x)=\frac{Y_{1}-Y_{0}}{X_{1}-X_{0}}\left(X-X_{0}\right),
$$

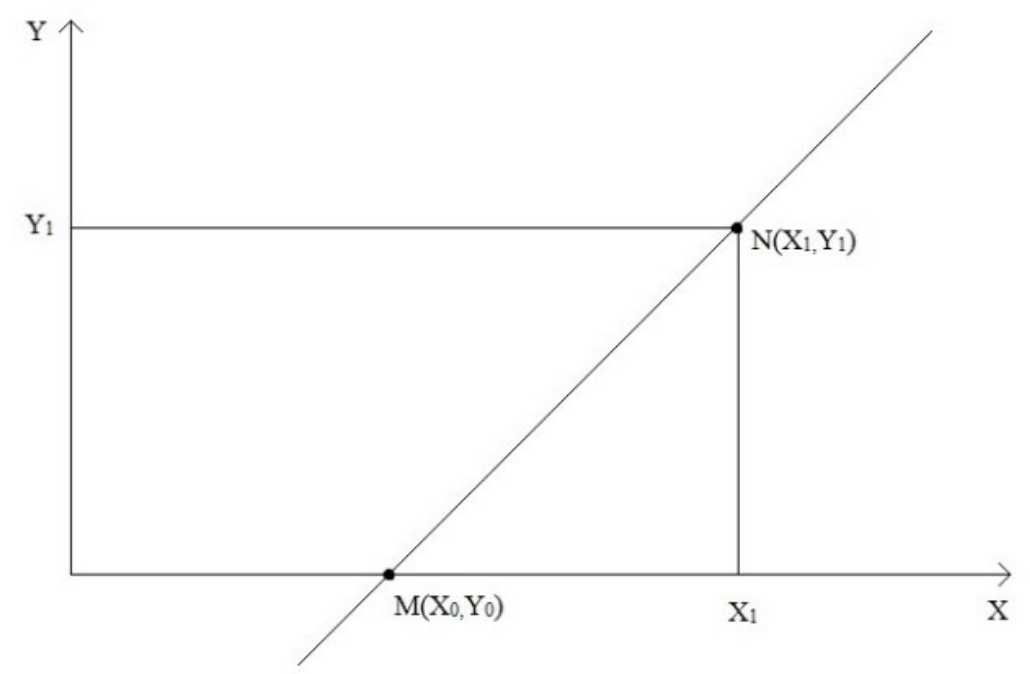

Figure 2. Function of describing the trajectory.

The block scheme of the movement is shown in Figure 3. And the full implementation of this method is shown in Algorithm 2.

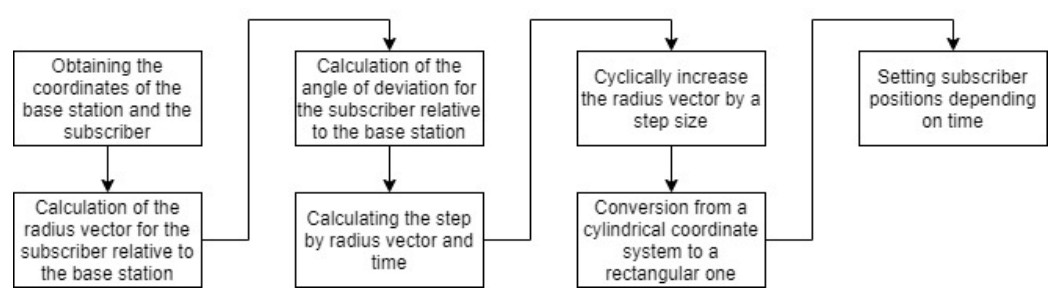

Figure 3. Block scheme of the movement.

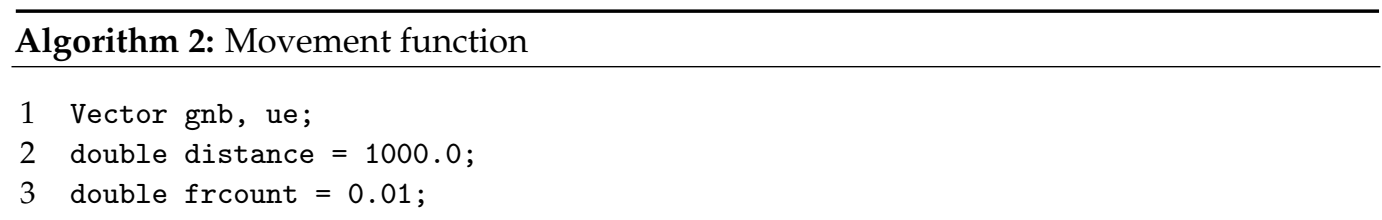




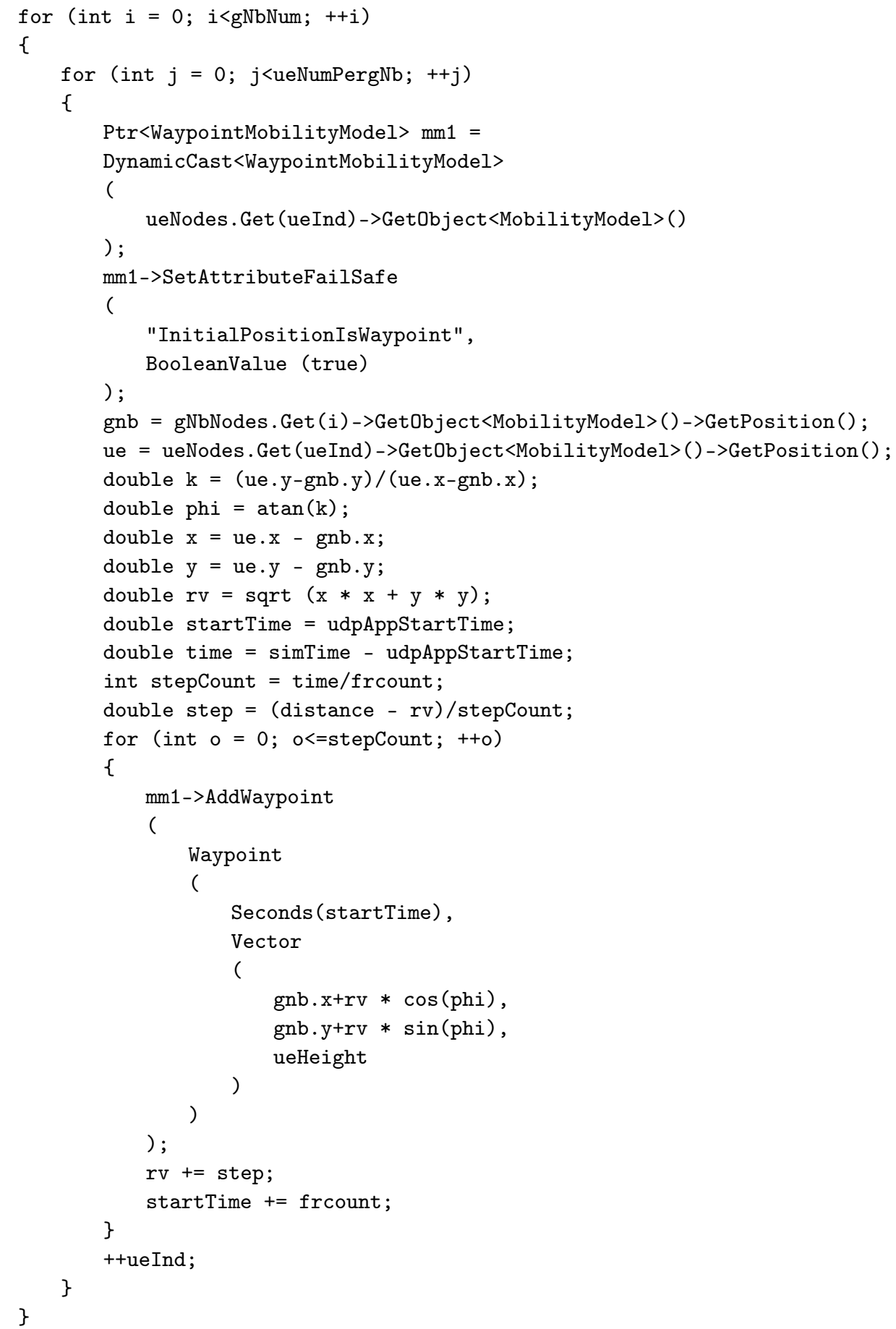

It was decided to perform a re-simulation with the replacement of the propagation loss model to check the functioning of the algorithms. The Okumura-Hata model was used when re-checking. This model was chosen due to different calculation principles from the 3GPP propagation loss model. From a practical point of view, this model is not applied for 5th generation networks, but its application is acceptable for the pursuance of the research [44].

\section{Results}

A simulation script was written using the developed algorithms for movement and distribution. There are two base stations in the scenario, and five subscriber nodes are 
placed around each of them. The distance range between base stations and nodes is 100 to $150 \mathrm{~m}$. The maximum removal distance is $1000 \mathrm{~m}$. When studying interference, a rather large contribution to its change is made by the power of the signal source and the path loss model. For both experiments, the power was $46 \mathrm{~dB}$. To compare the results obtained, it was decided to consider a scenario with the use of various models of signal propagation. Okumura and 3GPP were chosen as path loss models.

After completion of the distribution, the subscribers will have randomly located in an area of specified radius with the center at the base station location. The distribution result is shown in Figure 4.

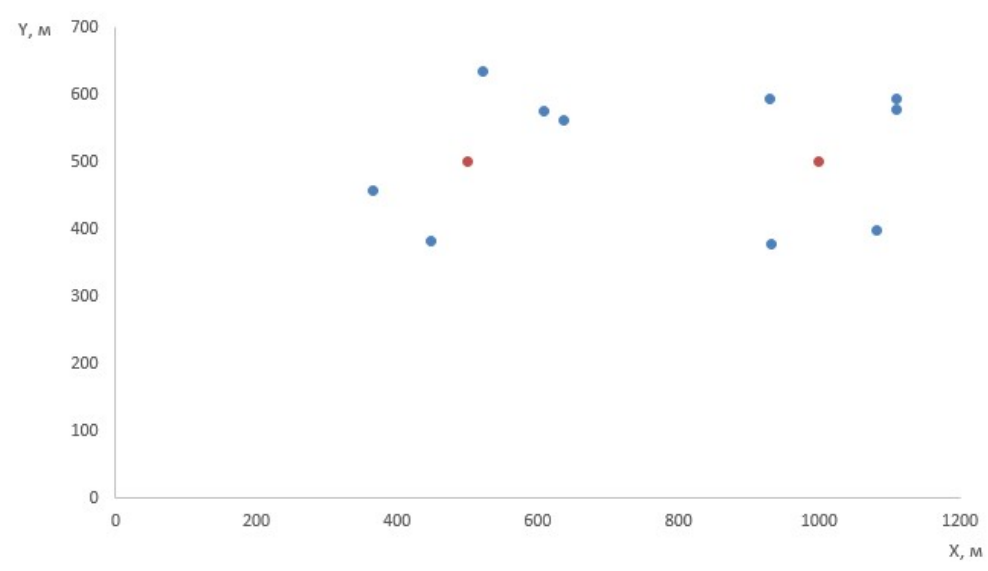

Figure 4. Result of distribution.

\subsection{Using 3GPP Propagation Loss Model}

A scenario that implements the transmission of URLLC traffic using the 3GPP propagation loss model was used during the experiment. The result of the distribution is presented in Table 2.

Based on the obtained results, it can be concluded that at the initial time, when 10 subscribers are distributed around two base stations at a distance of 100 to $150 \mathrm{~m}$, the SINR value varies from 22 to $50 \mathrm{~dB}$. This spread is due to the influence of interference. Additionally, there is no packet loss when data is transmitted since the BLER value remains unchanged. The obtained data were compared with simulation experiments and data from real-world equipment reviewed in [45]. Despite using different frequency bands, data obtained at the start time are similar in appearance in both works.

Table 2. Numerical result of the distribution.

\begin{tabular}{ccccc}
\hline BS Index & User Index & Distance & SINR & BLER \\
\hline 1 & 1 & 131.22 & 30.043 & 0 \\
1 & 2 & 135.79 & 33.904 & 0 \\
1 & 3 & 141.28 & 30.813 & 0 \\
1 & 4 & 149.75 & 33.342 & 0 \\
1 & 5 & 128.71 & 40.246 & 0 \\
2 & 6 & 117.00 & 24.318 & 0 \\
2 & 7 & 139.74 & 29.138 & 0 \\
2 & 8 & 130.33 & 22.595 & 0 \\
2 & 9 & 142.81 & 23.107 & 0 \\
2 & 10 & 133.53 & 49.786 & 0 \\
\hline
\end{tabular}

The Table 3 contains the results from one among 10 existing users obtained after running the previous simulation scenario where was added the ability of a subscriber to move away from the base station. Based on the obtained data, it can be seen that at a distance of less than $400 \mathrm{~m}$, the SINR parameter value is sufficient for sending all available packets because the BLER indicator $=0$. At a distance over $409 \mathrm{~m}$, the SINR indicator 
decreases, and the increase in the BLER value is inversely proportional. The distance from the base station exceeding $687.24 \mathrm{~m}$ brings the BLER indicator to one (100\%) that indicates a complete loss of data.

Table 3. Subscriber movement results.

\begin{tabular}{cccc}
\hline Time & Distance & SINR & BLER \\
\hline 540 & 252.85 & 24.785 & 0 \\
726 & 409.23 & 20.641 & 0.004 \\
810 & 487.42 & 20.109 & 0.19 \\
852 & 522.17 & 19.557 & 0.837 \\
1045 & 687.24 & 19.21 & 1 \\
\hline
\end{tabular}

\subsection{Using Okumura-Hata Propagation Loss Model}

The results obtained with the distribution of nodes are presented in the Table 4 . The distance values show that the algorithm functions correctly, and the result does not fall outside the range of 100-150 m. The SINR value differs from the values obtained using the 3GPP model. It is due to the fact that the initial positions of the subscribers are set randomly, and the distance for one pair is different. Additionally, the distribution model itself plays an important role. The total signal loss is different due to different calculation methods. While the SINR differs at the starting positions, the BLER value remains unchanged at 0 .

Table 4. Numerical result of the distribution.

\begin{tabular}{ccccc}
\hline BS Index & User Index & Distance & SINR & BLER \\
\hline 1 & 1 & 129.24 & 33.482 & 0 \\
1 & 2 & 137.68 & 31.921 & 0 \\
1 & 3 & 126.53 & 34.481 & 0 \\
1 & 4 & 131.73 & 34.883 & 0 \\
1 & 5 & 144.84 & 37.48 & 0 \\
2 & 6 & 130.81 & 30.751 & 0 \\
2 & 7 & 125.19 & 37.416 & 0 \\
2 & 8 & 146.3 & 35.166 & 0 \\
2 & 9 & 132.69 & 37.24 & 0 \\
2 & 10 & 105.02 & 42.076 & 0 \\
\hline
\end{tabular}

The results of the movement algorithm are presented in the Table 5. The model works correctly, and the subscriber moves away from the base station steady, in common with the experiment with 3GPP. The results show that the rate of degradation of a signal quality according to distance is higher as contrasted with the 3GPP propagation model. It is associated with the calculation principle of loss in Okumura-Hata. Due to such a sharp drop at a distance of $208.33 \mathrm{~m}$, an increase in BLER is already observed. The number of lost packets becomes more than $60 \%$ upon reaching $234.4 \mathrm{~m}$. The BLER value goes to 1 as the distance increases beyond $243.96 \mathrm{~m}$, which indicates the complete packet loss.

Table 5. Subscriber movement results.

\begin{tabular}{cccc}
\hline Time & Distance & SINR & BLER \\
\hline 471 & 191.05 & 27.008 & 0 \\
491 & 208.33 & 25.894 & 0.0006 \\
501 & 217.02 & 23.873 & 0.2483 \\
521 & 234.4 & 22.628 & 0.6202 \\
531 & 243.09 & 22.253 & 0.9987 \\
532 & 243.96 & 19.566 & 1.0000 \\
\hline
\end{tabular}




\section{Conclusions}

Network system designers need tools to simulate different situations. An analysis of the available ready-made solutions was carried out in this work. Additionally, a review of solutions was conducted directly associated with the chosen simulator. It was proposed the own implementation of two algorithms for distribution and movement based on this review, aimed to estimate the quality of the signal in various situations. The obtained data were compared with the results of other experiments. There was also a comparison with real data. The performed experiments show the correctly functioning of the algorithms, and, also, it is possible using loss models not intended for this type of communication. However, their capabilities are limited overmuch (the moment of total packet loss occurs at a shorter distance when using the Okumura-Hata loss model compared with the 3GPP model).

Author Contributions: Conceptualization, D.B. (Dmitry Baranov) and A.T.; methodology, D.B. (Dmitry Baranov) and A.T.; software, D.B. (Dmitry Baranov) and A.T.; validation, A.T., D.B. (Dmitry Bragin), and A.K.; formal analysis, A.T.; investigation, A.T.; resources, D.B. (Dmitry Bragin) and A.K.; data curation, A.T.; writing—original draft preparation, D.B. (Dmitry Baranov) and A.T.; writingreview and editing, D.B. (Dmitry Baranov); visualization, D.B. (Dmitry Baranov); supervision, D.B. (Dmitry Bragin); project administration, D.B. (Dmitry Bragin); funding acquisition, D.B. (Dmitry Bragin). All authors have read and agreed to the published version of the manuscript.

Funding: The article was prepared as part of implantation of the «Leading research center (LRC) «Trusted Sensor Systems», financial support provided by Ministry of Digital Development, Communications and Mass Media of the Russian Federation and Russian Venture Company (RVC JSC) (Agreement №009/20 dated 10 April 2020).

Data Availability Statement: No new data were created or analyzed in this study. Data sharing is not applicable to this article.

Conflicts of Interest: The authors declare no conflict of interest.

\section{Abbreviations}

The following abbreviations are used in this manuscript:

$\begin{array}{ll}\text { 3GPP } & \text { 3rd Generation Partnership Project } \\ \text { eMBB } & \text { Enhanced mobile broadband } \\ \text { mMTC } & \text { massive Machine Type Communication } \\ \text { NS3 } & \text { Network Simulator 3 } \\ \text { ULLRC } & \text { Ultra Low Latency Reliable Communication } \\ \text { GUI } & \text { Graphical user interface } \\ \text { OSI } & \text { The Open Systems Interconnection model } \\ \text { CORE } & \text { Common Open Research Emulator } \\ \text { SDN } & \text { Software-Defined Networking } \\ \text { MAC } & \text { Media Access Control } \\ \text { EPC } & \text { Evolved Packet System } \\ \text { LTE } & \text { Long-Term Evolution } \\ \text { NR } & \text { New Radio } \\ \text { QCN } & \text { Quantized Congestion Notification } \\ \text { FD-MIMO } & \text { Full Dimensional Multiple Input Multiple Output } \\ \text { SANSA } & \text { Shared Access Terrestrial-Satellite Backhaul Network enabled by Smart Antennas } \\ \text { SINR } & \text { Signal to Interference plus Noise Ratio } \\ \text { BLER } & \text { Block Error Rate } \\ \text { Phy } & \text { Physical } \\ \text { Doc. } & \text { Documentation }\end{array}$

\section{References}

1. Novokhrestov, A.; Konev, A.; Shelupanov, A. Model of threats to computer network software. Symmetry 2019, 11, 1506. [CrossRef]

2. Novokhrestov, A.; Konev, A. Mathematical model of threats to information systems. In Proceedings of the Prospects of Fundamental Sciences Development, Tomsk, Russia, 26-29 April 2016.

3. Aziz, N.N.A.; Rechie, R.M.A.; Bakry, B.B.M.; Rahman, R.A.; Yussoff, Y.M. Analysing Smart Home Security Using Packet Tracer Simulation Software. In Proceedings of the 2021 IEEE 11th IEEE Symposium on Computer Applications \& Industrial Electronics (ISCAIE), Penang, Malaysia, 3-4 April 2021; pp. 239-244. 
4. Trabelsi, Z.; Saleous, H. Exploring the Opportunities of Cisco Packet Tracer For Hands-on Security Courses on Firewalls. In Proceedings of the 2019 IEEE Global Engineering Education Conference (EDUCON), Dubai, United Arab Emirates, 8-11 April 2019; pp. 411-418.

5. Sjoholmsierchio, M.; Hale, B.; Lukaszewski, D.; Xie, G. Strengthening SDN Security: Protocol Dialecting and Downgrade Attacks. In Proceedings of the IEEE 7th International Conference on Network Softwarization, Tokyo, Japan, 28 June-2 July 2021 ; pp. 321-329.

6. Howard, W.; Borowczak, M. Detecting DDoS Attacks Near The Edge with Router Canaries. In Proceedings of the IEEE 10th International Conference on Consumer Electronics, Berlin, Germany, 9-11 November 2020; pp. 1-4.

7. Acosta, J.C.; Basak, A.; Kiekintveld, C.; Leslie, N.; Kamhoua, C. Cybersecurity Deception Experimentation System. In Proceedings of the IEEE Secure Development, Atlanta, GA, USA, 28-30 September 2020; pp. 34-40.

8. Liu, S.; Wang, H.; Liu, J.; Xian, M. Feasibility analysis of network security teaching platform based on KVM and GNS3. In Proceedings of the International Conference on Information Technology and Computer Application, Guangzhou, China, 20-22 December 2019; pp. 310-313.

9. Korniyenko, B.; Galata, L.; Ladieva, L. Research of Information Protection System of Corporate Network Based on GNS3. In Proceedings of the IEEE International Conference on Advanced Trends in Information Theory, Kyiv, Ukraine, 18-20 December 2019; pp. 244-248.

10. Yingshu, W.; Xu, W.; Yu, Z.; Qing, L.; Juanjuan, Z.; Shu, Y.; Fucai, Y. Review on Network Virtualization Simulation Software. J. Southwest Jiaotong Univ. 2020, 55, 34-40.

11. Masek, P.; Stusek, M.; Zeman, K.; Drapela, R.; Ometov, A.; Hosek, J. Implementation of 3GPP LTE Cat-M1 Technology in NS-3: System Simulation and Performance. In Proceedings of the 11th International Congress on Ultra Modern Telecommunications and Control Systems and Workshops, Dublin, Ireland, 28-30 October 2019; pp. 1-7.

12. ns3. Available online: https://www.nsnam.org/ (accessed on 11 September 2021).

13. Mezzavilla, M.; Dutta, S.; Zhang, M.; Akdeniz, M.R.; Rangan, S. 5G mmWave Module for ns-3 Network Simulator. In Proceedings of the 18th ACM International Conference on Modeling, Analysis and Simulation of Wireless and Mobile Systems, Cancun, Mexico, 2-6 November 2015; pp. 283-290.

14. Mezzavilla, M.; Zhang, M.; Polese, M.; Ford, R.; Dutta, S.; Rangan, S.; Zorzi, M. End-to-End Simulation of 5G mmWave Networks. IEEE Commun. Surv. Tutor. 2018, 20, 2237-2263. [CrossRef]

15. Ford, R.; Zhang, M.; Dutta, S.; Mezzavilla, M.; Rangan, S.; Zorzi, M. A Framework for End-to-End Evaluation of 5G mmWave Cellular Networks in ns-3. In Proceedings of the Workshop on ns-3, Seattle, WA, USA, 15-16 June 2016; pp. 85-92.

16. Abdulrazzak, F.; Abdulaziz, E.; Al-Hussaini, K. Performance Analysis for TCP Protocols over mm Wave in 5G Cellular Networks. In Proceedings of the First International Conference of Intelligent Computing and Engineering, Hadhramout, Yemen , 15-16 Decemebr 2019; pp. 1-6.

17. Herranz, C.; Martín-Sacristán, D.; Monserrat, J.F.; Zhang, M.; Rangan, S.; Mezzavilla, M. A 3GPP NR compliant beam management framework to simulate end-to-end mmwave networks. In Proceedings of the 21st ACM International Conference on Modeling, Analysis and Simulation of Wireless and Mobile Systems, Montreal, QC, Canada, 28 October-2 November 2018 ; pp. 119-125.

18. Polese, M.; Zorzi, M.; Mezzavilla, M.; Rangan, S. Mobility management for TCP in mmWave networks. In Proceedings of the 1st ACM Workshop on Millimeter-Wave Networks and Sensing System, Snowbird, UT, USA, 16 October 2017; pp. 11-16.

19. Lavacca, F.G.; Salvo, P.; Costantini, L.; Mammi, E.; Pompei, S.; Rea, L.; Teodori, M. Studying and Simulation of a NS3 frameworktowards a $5 \mathrm{G}$ Complete Network Platform. In Proceedings of the International Workshop on Fiber Optics in Access Networks, Sarajevo, Bosnia and Herzegovina, 2-4 September 2019; pp. 62-67.

20. Bojovic, B.; Lagen, S.; Giupponi, L. Implementation and evaluation of frequency division multiplexing of numerologies for 5G new radio in ns-3. In Proceedings of the 10th Workshop on ns-3, Surathkal, India, 13-14 June 2018; pp. 37-44.

21. Patriciello, N.; Lagen, S.; Giupponi, L.; Bojovic, B. The Impact of NR Scheduling Timings on End-to-End Delay for Uplink Traffic. In Proceedings of the IEEE Global Communications Conference, Waikoloa, HI, USA, 9-13 December 2019; pp. 1-6.

22. Paramonov, A.; Peng, J.; Kashkarov, D.; Muthanna, A.; Elgendy, I.A.; Koucheryavy, A.; Maleh, Y.; El-Latif, A.; Ahmed, A. Study and Analysis of Multiconnectivity for Ultrareliable and Low-Latency Features in Networks and V2X Communications. Wirel. Commun. Mob. Comput. 2021, 2021, 1718099. [CrossRef]

23. Sen, A.; Mondal, A.; Palit, B.; Jayatheerthan, J.; Paul, K.; Chakraborty, S. Sandip Chakraborty. An ns3-based Energy Module of 5G NR User Equipments for Millimeter Wave Networks. In Proceedings of the IEEE Conference on Computer Communications Workshops, Vancouver, BC, Canada, 10-13 May 2021; pp. 1-2.

24. Ateya, A.A.A.; Muthanna, A.; Kirichek, R.; Hammoudeh, M.; Koucheryavy, A. Energy- and Latency-Aware Hybrid Offloading Algorithm for UAVs. IEEE Access 2019, 7, 37587-37600. [CrossRef]

25. Zeman, K.; Masek, P.; Stusek, M.; Hosek, J.; Silhavy, P. Accuracy comparison of propagation models for mmWave communication in NS-3. In Proceedings of the 9th International Congress on Ultra Modern Telecommunications and Control Systems and Workshops, Munich, Germany , 6-8 November 2017; pp. 334-340.

26. Patriciello, N.; Lagen, S.; Giupponi, L.; Bojovic, B. An Improved MAC Layer for the 5G NR ns-3 Module. In Proceedings of the 2019 Workshop on ns-3, Florence, Italy, 19-20 June 2019; pp. 41-48. 
27. Ferreira, G.; Barreto, P.S.; Caetano, M.F.; Alchieri, E.; Vartiainen, J.; Karvonen, H.; Matinmikko-Blue, M.; Seki, J. A Tool for Developing Collaborative Sensing and Cognitive MAC Layer Solutions for 5G in Rural Areas. In Proceedings of the 16th International Symposium on Wireless Communication Systems, Oulu, Finland, 27-30 August 2019; pp. 58-62.

28. Sun, Y.; Jiang, L.; He, C.; He, D.; Li, P. Stable and Fair Quantized Notification for 5G Mobile Network. In Proceedings of the IEEE International Symposium on Circuits and Systems, Sapporo, Japan, 26-29 May2019; pp. 1-5.

29. Nishanthi, C.H.; Ramamurthy, N. Improving spectral efficiency and low latency in 5G framework utilizing FD-MIMO. J. Ambient. Intell. Humaniz. Comput. 2021, 12, 6955-6967. [CrossRef]

30. Jayalakshmi, S.; Sangeetha, N.; Swetha, S.; kumar, D.T.A. Network slicing and performance analysis of $5 \mathrm{~g}$ networks based on priority. Int. J. Sci. Technol. Res. 2019, 8, 3623-3627.

31. Diez, L.; Fernández, A.; Khan, M.; Zaki, Y.; Agüero, R. Can We Exploit Machine Learning to Predict Congestion over mmWave 5G Channels? Appl. Sci. 2020, 10, 6164. [CrossRef]

32. Liu, Y.; He, W.; Wang, Y.; Yang, H. Network-Assisted Neural Adaptive Naked-Eye 3D Video Streaming Over Wireless Networks. IEEE Access 2019, 7, 141363-141373. [CrossRef]

33. Murudkar, C.V.; Gitlin, R.D. Optimal-Capacity, Shortest Path Routing in Self-Organizing 5G Networks using Machine Learning. In Proceedings of the IEEE 20th Wireless and Microwave Technology Conference, Cocoa Beach, FL, USA, 8-9 April 2019; pp. 1-5.

34. Vaishnavi, C.; Ashok Kumar, A.R.; Selvakumar, G.; Chaudhari, S.Y. Self Organizing Networks Coordination Function between Intercell Interference Coordination and Coverage and Capacity Optimisation using Support Vector Machine. In Proceedings of the International Conference on Intelligent Computing and Control Systems, Madurai, India, 15-17 May 2019 ; pp. 316-320.

35. Lavacca, F.G.; Salvo, P.; Ferranti, L.; Speranza, A.; Costantini, L. Performance Evaluation of 5G Access Technologies and SDN Transport Network on an NS3 Simulator. Computers 2020, 9, 43. [CrossRef]

36. Giuntini, M.; Grazioso, P.; Matera, F.; Valenti, A.; Attanasio, V.; Di Bartolo, S.; Nastri, E. Enabling Optical Network Test Bed for 5G Tests. Fiber and Integrated Optics. Fiber Integr. Opt. 2016, 36, 3-24. [CrossRef]

37. Baranda, J.; Pascual, I.; Requena, M.; Mangues-Bafalluy, J. Deploying a containerized ns-3/LENA-based LTE mobile Network Service through the 5G-TRANSFORMER platform. In Proceedings of the IEEE Conference on Network Function Virtualization and Software Defined Networks, Verona, Italy, 27-29 November 2018; pp. 1-2.

38. Venkateswararao, K.; Swain, P.; Christophorou, C.; Pitsillides, A. Dynamic selection of virtual small base station in 5G ultra-dense network using initializing matching connection algorithm. In Proceedings of the IEEE International Conference on Advanced Networks and Telecommunications Systems, Goa, India , 16-19 December 2019; pp. 1-6.

39. Miozzo, M.; Bartzoudis, N.; Requena, M.; Font-Bach, O.; Harbanau, P.; López-Bueno, D.; Payaró, M.; Mangues, J. SDR and NFV extensions in the ns-3 LTE module for 5G rapid prototyping. In Proceedings of the IEEE Wireless Communications and Networking Conference, Barcelona, Spain, 15-18 April 2018; pp. 1-6.

40. Wu, B.; Ge, L.; Zeng, J.; Zheng, X.; Kuang, Y.; Su, X.; Wang, J. An Innovative EPC with Not Only Stack for beyond 5G Mobile Networks. In Proceedings of the IEEE 87th Vehicular Technology Conference, Porto, Portugal, 3-6 June 2018; pp. 1-5.

41. Patriciello, N.; Lagen, S.; Bojovic, B.; Giupponi, L. An E2E simulator for 5G NR networks. Simulation Modelling Practice and Theory. Simul. Model. Pract. Theory 2019, 96, 101933. [CrossRef]

42. Cornejo, A.; Landeros, S. Simulation Model and Comparison for Satellite Links in Ku and Ka Bands for Standards-based on DVB-S2 and NS3. IEEE Lat. Am. Trans. 2016, 14, 1689-1697. [CrossRef]

43. Artiga, X.; Nunez-Martinez, J.; Perez-Neira, A.; Vela, G.J.L.; Garcia, J.M.F.; Ziaragkas, G. Terrestrial-satellite integration in dynamic 5G backhaul networks. In Proceedings of the 8th Advanced Satellite Multimedia Systems Conference and the 14th Signal Processing for Space Communications Workshop, Palma de Mallorca, Spain, 5-7 September 2016; pp. 1-6.

44. Jakborvornphan, S. Analysis of path loss propagation models in mobile communication. J. Theor. Appl. Inf. Technol. 2020, 98, 725-730.

45. Banday, Y.; Rather, G.M.; Begh, G.R. SINR Analysis and Interference Management of Macrocell Cellular Networks in Dense Urban Environments. Wirel. Pers. Commun. 2020, 111, 21. [CrossRef] 